HD-THEP-98-02

\title{
Hopping Parameter Series Construction for Models with Nontrivial Vacuum
}

\author{
Thomas Reisz* \\ Institut für Theoretische Physik, Universität Heidelberg, \\ Philosophenweg 16, D-69120 Heidelberg, Germany
}

November 15, 2017

\begin{abstract}
Hopping parameter expansions are convergent power series. Under general conditions they allow for the quantitative investigation of phase transition and critical behaviour. The critical information is encoded in the high order coefficients. Recently, 20th order computations have become feasible and used for a large class of lattice field models both in finite and infinite volume. They have been applied to quantum spin models and field theories at finite temperature. The models considered are subject to a global $\mathbf{Z}_{2}$ symmetry or to an even larger symmetry group such as $O(N)$ with $N \geq 2$.

In this paper we are concerned with the technical details of series computations to allow for a nontrivial vacuum expectation value $\langle\rho(x)>\neq 0$, which is typical for models that break a global $\mathbf{Z}_{2}$ symmetry. Examples are scalar fields coupled to an external field, or manifestly gauge invariant effective models of Higgs field condensates in the electroweak theory, even in the high temperature phase. A nonvanishing tadpole implies an enormous proliferation of graphs and limits the graphical series computation to the 10th order. To achieve the hopping parameter series to comparable order as in the $\mathbf{Z}_{2}$ symmetric case, the graphical expansion is replaced by an expansion into new algebraic objects called vertex structures. In this way the 18th order becomes feasible.
\end{abstract}

\footnotetext{
*Supported by a Heisenberg Fellowship, Email address reisz@thphys.uni-heidelberg.de
} 


\section{Introduction}

Linked cluster expansions (LCE) provide a way to construct hopping parameter or high temperature series of correlation functions for a large class of lattice models [1, 2]. In contrast to standard perturbation theory, the series are convergent power series (for a recent proof cf. [3, 田 and references therein). Under general conditions the domain of convergence extends to the phase boundary. The nature of the transition such as its location, the order and the critical exponents, are encoded in the high order behaviour of the expansion coefficients.

In the recent past, much effort has been done to prolong the series for various field models on the lattice [5]-[10]. Fast and efficient methods have been developed such that susceptibilities and renormalized coupling constants are available up to the order 20 in the hopping parameter. In particular, they have been generated for lattice field theories at finite temperature [6] or quantum spin models. Particular applications concern the universality classes of high temperature transitions for $\mathrm{O}(\mathrm{N})$-symmetric field theories [7] and Gross-Neveu models, related to the QCD chiral phase transition [11]. More subtle questions concerning the applicability of dimensional reduction have been answered. The series have been generated and used in finite volume together with finite size scaling analysis to resolve the order of phase transitions [12].

The hopping parameter expansion amounts to a graphical representation of correlation functions. Appropriate graph classes have to be defined and generated, and their weights have to be computed. To make high order computation feasible, graphs are represented algebraically by incidence matrices. This representation needs to be one-to-one and fast [6].

To achieve high orders, some properties of the model and of the lattice are taken into account in the course of the graph construction. Beyond others, it is used that the models considered are subject to a global $\mathbf{Z}_{2}$ symmetry or a symmetry group including $\mathbf{Z}_{2}$ as a subgroup. This implies that only even correlation functions are non-trivial.

There are interesting models both in statistical mechanics and in quantum field theory where this global $\mathbf{Z}_{2}$ symmetry is absent on physical grounds or by explicit breaking. We give two examples.

- A scalar field theory on the lattice $\Lambda$, coupled to a nonvanishing external field,

$$
S(\Phi)=-\kappa \sum_{x, y \in \Lambda}^{\prime} \Phi(x) \Phi(y)+\sum_{x \in \Lambda}\left(\mu \Phi(x)^{2}+\lambda\left(\Phi(x)^{2}\right)^{2}\right)+H \sum_{x \in \Lambda} \Phi(x),
$$

where the prime denotes nearest neighbour interaction, and $H \neq 0$. Putting the hopping parameter $\kappa$ to its critical value $\kappa_{c}(\mu, \lambda, H=0)$ and letting $H \rightarrow 0$, the critical exponent $\delta$ is determined by the behaviour of $\langle\Phi(x)\rangle$. Knowledge of its series expansion in $\kappa$ allows for a high precision measurement of $\delta$. 
- Effective models in which the fluctuation fields are confined in value to some subset of the real numbers. An example is provided by the gauge invariant $\mathrm{SU}(2)$ Higgs model. At finite temperature this model is of great importance for the physics of the early universe. The high temperature phase transition is highly non-perturbative in the region of realistic Higgs masses. On a finite temperature lattice $\Lambda$, integration of the compact gauge fields without imposing a gauge fixing generates an effective model that is described by a partition function of the form 13

$$
Z=\int_{0}^{\infty} \prod_{x \in \Lambda}\left(d \xi(x) \xi(x)^{3}\right) \exp \left(-V_{e f f}(\xi)\right) .
$$

The Higgs field condensates and their correlations are obtained as correlation functions of the field $\xi(x)$. Although both the measure and the action $V_{\text {eff }}$ are formally invariant under the global $\mathbf{Z}_{2}$ transformation $\xi(x) \rightarrow-\xi(x)$, the field $\xi$ is confined to non-negative values, which implies that $\langle\xi(x)>\neq 0$ even in the high temperature phase.

In this paper we generalize the linked cluster expansion to models in which the $\mathbf{Z}_{2}$ symmetry is explicitly broken or does not exist at all. There are mainly two generalizations to be included. First, correlation functions with an odd number of fields do no more vanish in general. In turn, vertices are allowed to have odd number of lines attached. This is easily incorporated into the known algorithm and requires only a slight generalization, although the number of graphs is considerably enhanced. Second, as the more subtle point, the vacuum expectation value of the field, the tadpole moment, does no more vanish in general,

$$
<\rho(x)>\neq 0 .
$$

This property requires the introduction of new graph classes with different topological properties. For instance, one-particle irreducible (1PI) correlation functions, represented as a sum over 1PI graphs, play an important role in the course of the expansion. Once (3) holds, however, 1PI refers only to external line channels. The necessary modifications will be given here.

Furthermore, the combinatorical complexity is drastically enhanced. We expect that without further improvement the complexity to 10th order is already as much as the 20th order before.

To achieve comparable order as in the $\mathbf{Z}_{2}$ symmetric case (order 18-20), we replace where required the concept of graphs by the concept of vertex structures. Vertex structures have already been introduced in [6]. They were used mainly to avoid a recomputation from scratch of LCE series if the coupling constants of the model are changed. Using them also avoids severe roundoff errors due to the finite precision of real coupling constants.

Here, in addition we use vertex structures to avoid the graphical construction of the tadpoles and the combinatorical problems and computational limitations related 
to them. In a way, we can view a vertex structures as an equivalence class in the set of graphs considered so that every graph belongs to (precisely) one vertex structure, and such that

- the graphical details that are not needed for further computations are hidden, and

- equivalence classes are as large as possible, that is, combine as many graphs as possible. The number of vertex structures then becomes rather small.

Summmation over graphs then is replaced by summation over vertex structures, with appropriate weights. We intent to make the set of vertex structures an algebra with operations that have their analog for graphs (such as concatenation), but otherwise are much more efficient and fast. In this algebra the series of tadpoles and the correlation functions are constructed.

Once we know this structure it is straightforward to implement the construction of the series expansions in a language like $\mathrm{C}$ or $\mathrm{C}++$.

In Sect. 2 we recapitulate the principles of the linked cluster expansion in graphical terms. This is done only to the extent required to understand the necessary modifications for non-zero vacuum expectation values. We will show that this is conveniently implemented by a second kind of vertex renormalization, supplemented by a recurrence relation for computing the tadpole moments. In order to obtain comparable efficiency and order as in the symmetric case, in Sect. 3 the algebra of weighted vertex structures is introduced. The hopping parameter series construction then is done in this algebra, generalizing the graphical expansion to a vertex structure expansion. A summary is given in Sect. 4.

\section{The graphical approach to series construction.}

\subsection{Principles}

We start with a short survey of the series construction. We put particularly emphasis on those points important to understand the differences of models with nonvanishing vacuum expectation value. For many details that are the same as for symmetric models and that are not repeated here, the reader is referred to [6].

Let $\Lambda$ denote a $D$-dimensional hypercubic lattice. It is assumed to be of shape $L_{0} \times L_{1} \times \cdots \times L_{D-1}$, with the $L_{i} \geq 4$ even, finite or infinite, and periodic boundary conditons imposed in the directions of finite length. We discuss models described by a partition function of the form

$$
\exp (W(J, v))=Z(J, v)=\int_{-\infty}^{\infty} \prod_{x \in \Lambda} d \mu(\rho(x)) \exp \left(-S_{h o p}(\rho, v)+\sum_{x \in \Lambda} J(x) \rho(x)\right),
$$


where $\rho$ denotes a real valued scalar field, and $J$ are external sources used to generate the correlation functions. The action $S_{h o p}$ is assumed to be of the form

$$
S(\rho, v)=-\frac{1}{2} \sum_{x \neq y \in \Lambda} \rho(x) v(x, y) \rho(y) .
$$

For pure nearest neighbour interaction,

$$
v(x, y)=\left\{\begin{aligned}
2 \kappa & , \quad x=y \pm \widehat{\nu} \text { for some } \nu \\
0 & , \quad \text { otherwise }
\end{aligned}\right.
$$

with $\widehat{\nu}$ the unit vector in the positive $\nu$ th direction, $\nu=0, \ldots, D-1$. We confine attention to this case, although more general pair interactions are feasible [4]. The single site measure $d \mu(\rho)$ is appropriately bounded for the partition function to exist and to be analytic for small hopping interaction $v(x, y)$ and small external sources $J(x)$. For instance, let $I$ be some real interval, finite or infinite, and $\chi_{I}$ the characteristic function of $I$,

$$
\chi_{I}(\rho)=\left\{\begin{array}{lll}
1 & , & \rho \in I \\
0 & , & \text { otherwise. }
\end{array}\right.
$$

Then the hopping parameter series discussed below are convergent for

$$
d \mu(\rho)=d \rho \chi_{I}(\rho) e^{-V(\rho)}
$$

with $V(\rho)$ bounded from below by

$$
V(\rho) \geq c \rho^{2}-d, \quad c>0
$$

cf. [3, 4]. The two examples given in the introduction correspond to the single site measures

$$
d \mu(\rho)=d \rho \exp \left(-\rho^{2}-\lambda\left(\rho^{2}-1\right)^{2}+H \rho\right)
$$

and

$$
d \mu(\rho)=d \rho \rho \chi_{[0, \infty[}(\rho) \exp (-V(\rho))
$$

(where $\rho=\xi^{2}$, cf. (2)), with $V(\rho)$ bounded according to (9). Connected correlation functions are obtained by differentiation of the generating functional,

$$
W^{(n)}\left(x_{1}, \ldots, x_{n} \mid v\right)=<\rho\left(x_{1}\right) \cdots \rho\left(x_{n}\right)>^{c}=\left.\frac{\partial^{n}}{\partial J\left(x_{1}\right) \cdots \partial J\left(x_{n}\right)} W(J, v)\right|_{J=0} .
$$

Susceptibilities are defined as zero momentum correlations such as

$$
\chi_{2}=\sum_{x \in \Lambda}<\rho(x) \rho(0)>^{c} ; \quad \mu_{2}=\sum_{x \in \Lambda}\left(\sum_{i=0}^{D-1} x_{i}^{2}\right)<\rho(x) \rho(0)>^{c} .
$$


Renormalized coupling constants are derived from them in a straightforward way.

The linked cluster expansion is the Taylor expansion of the generating functional $W(J, v)$ with respect to $v(x, y)$,

$$
W(J, v)=\left.\left(\exp \sum_{x, y \in \Lambda} v(x, y) \frac{\partial}{\partial \widehat{v}(x, y)}\right) W(J, \widehat{v})\right|_{\widehat{v}=0} .
$$

It generalizes in the obvious way to connected correlation functions. Multiple derivatives of $W$ with respect to $v(x, y)$ are managed by the identity [1]

$$
\frac{\partial W}{\partial v(x, y)}=\frac{1}{2}\left(\frac{\partial^{2} W}{\partial J(x) \partial J(y)}+\frac{\partial W}{\partial J(x)} \frac{\partial W}{\partial J(y)}\right)
$$

Correlation functions become series in the hopping parameter $\kappa$, with coefficients depending on the toplogy of the lattice and the interaction $\mu$, Eqn.(8).

The evaluation of the Taylor expansion rapidly becomes complex with increasing order, which is the number of derivatives with respect to $v(x, y)$. To manage it one is led in a natural way to a graphical device. In the present context, a graph is a strucutre

$$
\Gamma=\left(\mathcal{L}_{\Gamma}, \mathcal{B}_{\Gamma}, E_{\Gamma}, \Phi_{\Gamma}\right)
$$

where $\mathcal{L}_{\Gamma}$ and $\mathcal{B}_{\Gamma} \neq \emptyset$ are disjoint sets, the set of internal lines and the set of vertices of $\Gamma$, respectively. $E_{\Gamma}$ is a map

$$
E_{\Gamma}: \mathcal{B}_{\Gamma} \rightarrow\{0,1,2, \ldots\}, \quad u \rightarrow E_{\Gamma}(u),
$$

that assigns to every vertex $u \in \mathcal{B}_{\Gamma}$ the number of external lines $E_{\Gamma}(u)$ attached to it. If $E_{\Gamma}(u) \neq 0, u$ is called an external vertex of $\Gamma$. The number of external lines of $\Gamma$ is given by $\sum_{u \in \mathcal{B}_{\Gamma}} E_{\Gamma}(u)$. Finally, $\Phi_{\Gamma}$ is the incidence relation that assigns internal lines to their two endpoint vertices. Selflines are excluded. We consider lines as being unoriented, so $\Phi_{\Gamma}$ maps onto unoriented pairs of vertices

$$
\Phi_{\Gamma}: \mathcal{L}_{\Gamma} \rightarrow \overline{\left(\mathcal{B}_{\Gamma} \times \mathcal{B}_{\Gamma}\right)} .
$$

where for $v, w \in \mathcal{B}_{\Gamma}$ we have $\overline{(v, w)}=\overline{(w, v)}$. For every pair $v, w \in \mathcal{B}_{\Gamma}, v \neq w$, the number of common lines of $v$ and $w$ is defined by

$$
m(v, w)=\Phi_{\Gamma}^{-1}(\overline{(v, w)}) .
$$

The number of lines attached to $u \in \mathcal{B}_{\Gamma}$ is given by

$$
l(u):=\sum_{w \in \mathcal{B}_{\Gamma}} m(u, w)+E_{\Gamma}(u) .
$$

Connectedness is as usual path connectedness.

Two graphs

$$
\Gamma_{1}=\left(\mathcal{L}_{1}, \mathcal{B}_{1}, E_{1}, \Phi_{1}\right), \Gamma_{2}=\left(\mathcal{L}_{2}, \mathcal{B}_{2}, E_{2}, \Phi_{2}\right)
$$


are called (topologically) equivalent if there are two maps $\phi_{1}: \mathcal{B}_{1} \rightarrow \mathcal{B}_{2}$ and $\phi_{2}$ : $\mathcal{L}_{1} \rightarrow \mathcal{L}_{2}$, such that

$$
\Phi_{2} \circ \phi_{2}=\bar{\phi}_{1} \circ \Phi_{1}, \quad E_{2} \circ \phi_{1}=E_{1},
$$

where $\circ$ means decomposition of maps, and

$$
\bar{\phi}_{1}: \overline{\mathcal{B}_{1} \times \mathcal{B}_{1}} \rightarrow \overline{\mathcal{B}_{2} \times \mathcal{B}_{2}}, \quad \bar{\phi}_{1}(v, w)=\left(\phi_{1}(v), \phi_{1}(w)\right) .
$$

A symmetry of a graph $\Gamma=(\mathcal{L}, \mathcal{B}, E, \Phi)$ is a pair of maps $\phi_{1}: \mathcal{B} \rightarrow \mathcal{B}$ and $\phi_{2}: \mathcal{L} \rightarrow$ $\mathcal{L}$, such that

$$
\Phi \circ \phi_{2}=\bar{\phi}_{1} \circ \Phi, \quad E \circ \phi_{1}=E .
$$

The number of those maps is called the symmetry number of $\Gamma$.

The set of equivalence classes of connected graphs with $E$ external and $L$ internal lines is henceforth denoted by $\mathcal{G}_{E}(L)$, and

$$
\mathcal{G}_{E}:=\bigcup_{L \geq 0} \mathcal{G}_{E}(L) .
$$

Further graph classes will be introduced when they are needed below. Graphical expansions are done with respect to equivalence classes of graphs. In the following a graph $\Gamma$ is understood to represent the complete class of graphs equivalent to $\Gamma$ in the sense above.

Connected correlation functions get a representation as a sum over connected graphs, each graph being endowed with the appropriate weight. In this description a line of a graph represents a hopping propagator. For instance, the 2-point susceptibility becomes

$$
\begin{aligned}
\chi_{2} & =\sum_{L \geq 0}(2 \kappa)^{L} \sum_{\Gamma \in \mathcal{G}_{2}(L)} a(\Gamma), \\
a(\Gamma) & =w(\Gamma) \prod_{u \in \mathcal{B}_{\Gamma}} \stackrel{\circ c}{v}_{l(u)}(\mu),
\end{aligned}
$$

where

$$
{\stackrel{\circ}{v_{i}}}_{i}(\mu)=W^{(i)}(0, \ldots, 0 \mid v=0) .
$$

$w(\Gamma)$ is a rational number that accounts for the various topological symmetry and lattice imbedding numbers of $\Gamma$. The couplings $\stackrel{\circ}{v_{i}^{c}}(\mu)$ are real numbers and depend only on the local coupling constants of the model, that is on the single link measure $\mu$. They are obtained by numerical integration.

The computation of correlation functions proceeds by constructing more restricted and hence smaller graph classes. They are then composed of the latter, either analytically or by concatenation. The first step is the introduction of 1-particle irreducible (1PI) graphs. A graph is called 1PI if it has the following property: 
Remove a single line. The resulting graph then has only one connected component which has external lines attached. For instance, the 2-point susceptibility $\chi_{2}$ becomes

$$
\chi_{2}=\frac{\chi_{2}^{1 P I}}{1-(2 \kappa)(2 D) \chi_{2}^{1 P I}},
$$

in graphical terms

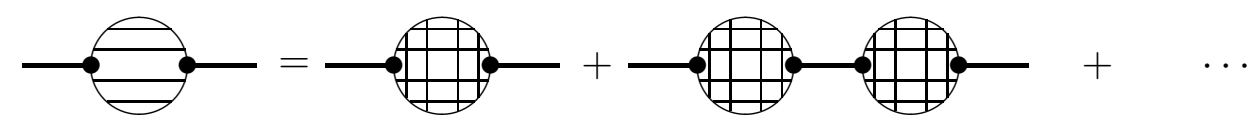

with

$$
\chi_{2}^{1 P I}=\sum_{L \geq 0}(2 \kappa)^{L} \sum_{\Gamma \in \mathcal{G}_{2}^{1 P I}(L)} a(\Gamma)
$$

that is, $\chi_{2}^{1 P I}$ is composed of 1PI graphs only.

The second necessary step is to compose 1PI graphs of the even more restricted classes of 1-vertex irreducible (1VI) graphs and renormalized moments. This is called vertex renormalization [1]. A graph $\Gamma$ is called $1 \mathrm{VI}$ if the following condition is satisfied. Remove an arbitrary vertex $u$ of $\Gamma$, together with the external and internal lines attached to $u$. We denote the resulting graph by $\Gamma_{u}$. Then, every connected component of $\Gamma_{u}$ has at least one external line left attached to one of its vertices. We write

$$
\mathcal{S}_{k}(L)=\left\{\Gamma \in \mathcal{G}_{k}^{1 \mathrm{PI}}(L) \mid \Gamma \text { is } 1 \mathrm{VI}\right\}
$$

for the set of graphs that are both 1PI and 1VI. On the other hand, renormalized moment diagrams are 1PI graphs that have exactly one external vertex,

$$
\widehat{\mathcal{Q}}_{k}(L)=\left\{\Gamma \in \mathcal{G}_{k}^{1 \mathrm{PI}}(L) \mid \Gamma \text { has } 1 \text { and only } 1 \text { external vertex }\right\} .
$$

The reason for the ${ }^{\wedge}$ will become clear in the next section. With both of these notions, susceptibilities are represented as

$$
\chi_{E}^{1 \mathrm{PI}}=\sum_{L \geq 0}(2 \kappa)^{L} \sum_{\Gamma \in \mathcal{S}_{E}(L)} a(\Gamma),
$$

where the weights $a(\Gamma)$ are computed in the same way as before by (27), with the following exception only. The vertex couplings $\stackrel{\circ}{v}_{i}^{c}(\mu)$ therein are replaced by the renormalized moments

$$
{\stackrel{\circ}{v_{i}}}_{i}(\mu) \rightarrow v_{i}^{c}(\kappa, \mu)=\sum_{L \geq 0}(2 \kappa)^{L} \sum_{\Gamma \in \widehat{\mathcal{Q}}_{i}(L)} w(\Gamma) \prod_{u \in \mathcal{B}_{\Gamma}} \stackrel{\circ}{v}_{l(u)}^{c}(\mu) .
$$

1VI and renormalized moments provide a kind of product representation of 1PI graphs by 1VI diagrams and renormalized moments. In the final end, after having 
determined all the weights of the graphs, the coefficients are reorganized to give the series of $\chi_{E}^{1 P I}$.

Finally, we need to construct all of $\mathcal{S}_{k}$ and $\widehat{\mathcal{Q}}_{k}$. They are generated from a sequence of appropriate base classes. Also, in order to do the graph generation on the computer, we need an algebraic representation of graphs. This is primarily done by so-called incidence matrices. The representation needs to be one-to-one and efficient, that is, fast algorithms are required to identify graphs by putting them into "canonical" representations. These problems are solved such that for $\mathrm{O}(\mathrm{N})$ symmetric models the most important series are available to 20 th order in the hopping parameter.

There are fast algorithms to generate 1-line irreducible (1LI) graphs. A graph is called 1LI if it cannot be divided in two by cutting a single line. For models with vanishing vacuum expectation value $\langle\rho\rangle=0$, a graph is $1 \mathrm{LI}$ if and only if it is 1PI, so the notions 1LI and 1PI are equivalent for them. This concerns the graph classes $\mathcal{S}_{k}$ and $\widehat{\mathcal{Q}}_{k}$.

However, if the expectation value of the field $\langle\rho\rangle \neq 0$, the properties of being 1LI and being 1PI are no more the same. 1LI implies 1PI, but not vice versa. The classes of renormalized moments $\widehat{\mathcal{Q}}_{k}$ become enormously enhanced. This problem is addressed in the next subsection.

\subsection{Tadpoles}

Now suppose that the model under consideration is not subject to a $\mathbf{Z}_{2}$ symmetry. We consider the necessary modifications of the the series construction.

- Correlation functions with an odd number of fields do no more vanish in general. This implies that higher connected correlation functions have a more complicated representation in terms of 1PI ones.

- Graphs are no more restricted to having vertices only with an even number of lines attached. "Odd" vertices are allowed now.

- The vacuum expectation value of the field does no more vanish in general,

$$
\chi_{1}=<\rho(x)>\neq 0 .
$$

The first two generalizations are easily included. First, the map between connected and 1PI correlations is done in closed analytic form. We are still mainly concerned with 1PI graphs only. Furthermore, vertices with odd number of lines do not provide a major problem. The inclusion of $1 \mathrm{LI}$ graphs having vertices with an odd number of lines attached implies an enormous enhancement of their numbers. For instance, the graph class $\mathcal{S}_{2}(L)$ with number of internal lines $L \leq 18$ grows by about 3 orders of magnitude, as can be seen from Table 1 below. However, the 
algorithms are so efficient that they still work with this generalization. They require a minimal modification only, as long as 1LI graphs are concerned.

A non-vanishing one-point function (36) provides the major problem. Graphically we represent this expectation value by the "tadpole",

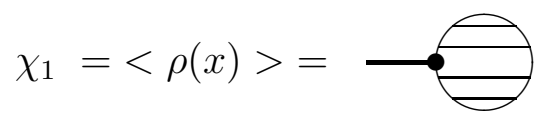

Now 1PI graphs are no more necessarily 1LI. In particular, by the very definition, tadpole graphs are always 1PI, but almost never 1LI. To obtain the graphical representation of $1 \mathrm{PI}$ n-point functions for $n \geq 2$ we have to

- find a way to construct the tadpole graphs, and

- attach the tadpoles to 1LI graphs in all possible ways.

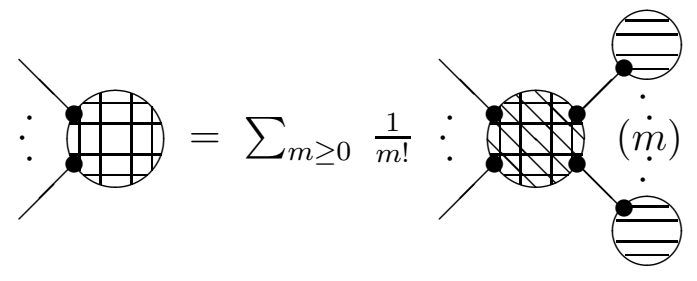

We can do better. In the last subsection we have recapitulated the composition of 1PI graphs by 1VI graphs and renormalized moments. Whereas the 1VI graphs of $\mathcal{S}_{k}$ are already 1LI, as follows from their definiton, the renormalized moments $\widehat{\mathcal{Q}}_{k}$ are $1 \mathrm{PI}$ only. Hence it is sufficient to do the above tadpole attachment only to generate $\widehat{Q}_{k}$. This amounts to a further vertex renormalization. We circumvent the construction of 1LI graphs with large number of external lines, which would be very expensive.

We define the sets of first renormalized moment graphs $\mathcal{Q}_{k}(L)$ by

$$
\mathcal{Q}_{k}(L)=\left\{\Gamma \in \mathcal{G}_{k}^{1 \mathrm{LI}}(L) \mid \Gamma \text { has } 1 \text { and only } 1 \text { external vertex }\right\}
$$

and the first renormalized moments themselves by

$$
\chi_{Q_{k}}=\sum_{L \geq 0}(2 \kappa)^{L} \sum_{\Gamma \in Q_{k}(L)} w(\Gamma) \prod_{u \in \mathcal{B}_{\Gamma}}^{\circ^{c}} v_{l(u)}(\mu) .
$$

The first renormalized moment graphs are 1LI and are constructed as for $\mathbf{Z}_{2}$ symmetric models. The second renormalized moments diagrams $\widehat{\mathcal{Q}}_{k}$, (33), are then obtained by attaching tadpoles to the vertices of the graphs of $\mathcal{Q}_{k}$. Let us assume 
for the moment that the series representation of the tadpole moment $\chi_{1}$ is known, for instance in the form

$$
\chi_{1}(\kappa, \mu)=\sum_{L \geq 0}(2 \kappa)^{L} \chi_{1}(L)
$$

For convenience we introduce the quantities

$$
\begin{aligned}
& \widehat{\chi}_{1}^{(1)}(\kappa, \mu)=\sum_{x \in \Lambda} v(x, 0) \chi_{1}(\kappa, \mu)=(2 \kappa)(2 D) \chi_{1}(\kappa, \mu), \\
& \widehat{\chi}_{1}^{(\nu)}(\kappa, \mu)=\frac{1}{\nu !}\left(\widehat{\chi}_{1}^{(1)}(\kappa, \mu)\right)^{\nu}=(2 \kappa)^{\nu} \frac{(2 D)^{\nu}}{\nu !}\left(\chi_{1}(\kappa, \mu)\right)^{\nu}, \quad \nu \geq 2 .
\end{aligned}
$$

With the tadpole vertices $v_{i}^{c, \operatorname{tadp}}(\kappa, \mu)$ defined by

$$
v_{i}^{c, \operatorname{tadp}}(\kappa, \mu)=\stackrel{\circ}{v}_{i}^{c}(\mu)+\sum_{\nu \geq 1} \stackrel{\circ}{v}_{i+\nu}^{c}(\mu) \widehat{\chi}_{1}^{(\nu)}(\kappa, \mu)
$$

the second vertex renormalization (35) is replaced by

$$
{\stackrel{\circ}{v_{i}}}_{i}(\mu) \rightarrow v_{i}^{c}(\kappa, \mu)=\sum_{L \geq 0}(2 \kappa)^{L} \sum_{\Gamma \in \mathcal{Q}_{i}(L)} \widetilde{a}(\Gamma)
$$

with

$$
\widetilde{a}(\Gamma)=w(\Gamma) \prod_{u \in \mathcal{B}_{\Gamma}} v_{l(u)}^{c, \operatorname{tadp}}(\kappa, \mu) .
$$

Finally, after having determined all the weights of the graphs, the coefficients are reorganized as before to give the series of $\chi_{E}^{1 P I}$.

We have assumed that the hopping parameter series (41) of the 1-point function is known. Actually, this series representation is obtained by (44) itself for $i=1$. For, we have $v_{1}^{c}(\kappa, \mu)=\chi_{1}(\kappa, \mu)$, and $\widehat{\chi}_{1}^{(\nu)}(\kappa, \mu)=O\left(\kappa^{\nu}\right)$. Hence (42,45) becomes the recursion relation

$$
\chi_{1}(\kappa, \mu)=\sum_{L \geq 0}(2 \kappa)^{L} \sum_{\Gamma \in \mathcal{Q}_{1}(L)} w(\Gamma) \prod_{u \in \mathcal{B}_{\Gamma}}\left(\stackrel{\circ}{v}_{l(u)}^{c}(\mu)+\sum_{\nu \geq 1} \stackrel{\stackrel{o}{v}^{c}}{l(u)+\nu}(\mu) \widehat{\chi}_{1}^{(\nu)}(\kappa, \mu)\right),
$$

with initial condition $\chi_{1}(\kappa, \mu)=\stackrel{\circ}{v}_{1}^{c}(\mu)+O(\kappa)$. This recursion is solved order by order in $\kappa$. We need the first renormalized moments $Q_{1}(L)$ with one external line, consisting of 1LI graphs only.

In summary, the generation of the hopping parameter series of m-point functions to order $M$, say, according to the discussion above proceeds as follows.

1. The single site coupling constants $\stackrel{\circ}{v}_{i}^{c}(\mu)$ for $1 \leq i \leq m+M$ are computed. This amounts to the evaluation of a sequence of low-dimensional integrals. 
2. Construction of the graphs of $\mathcal{S}_{E}(L)$ for $E<=m$ and $L \leq M$, and of the first renormalized moments $\mathcal{Q}_{E}(L)$ for $E+L \leq m+M$, alltogether being 1LI, and their weight factors. This is done as for $\mathbf{Z}_{2}$ symmetric models. The only generalization here is that we allow for vertices having an odd number of lines attached. This is easily included.

3. The series of the tadpole moment $\chi_{1}$ are computed by the recursion relation (46), starting with $\chi_{1}(\kappa, \mu)=\stackrel{\circ}{v}_{1}^{c}(\mu)+O(\kappa)$. This requires the knowledge of the first renormalized moments $Q_{1}(L)$ for $L \leq M$, obtained in step 2 .

4. Once the series of $\chi_{1}$ are known to order $M$, we compute the second renormalized moments $v_{i}^{c}(\kappa, \mu)$ according to (43,45), by attaching the tadpole moment in all possible ways to the vertices of the first renormalized moment graphs (the first vertex renormalization). These second renormalized moments then are attached to the vertices of the the graphs of $\mathcal{S}_{E}(L)$ with $E \leq m$ and $L \leq M$ (the second vertex renormalization). In this way we obtains the series coefficients of the desired 1PI correlation functions. The final computation of the connected correlations is done analytically. Of course each of the steps above involves a sorting of the series coefficients according to their order in $\kappa$.

\section{Vertex structures.}

\subsection{Motivation}

As we have seen in the last section, the only graphs that are actually generated and operated on are always 1LI. Their numbers for the simplest classes are listed in Table 1. By the procedure outlined at the end of the last section, all the other moments and correlations are no more obtained as a graphical representation but directly as hopping parameter series by the two vertex renormalizations.

Although this construction works in principle, there is need for improvement. This is for the following reasons.

- Beyond exceptional cases, in most applications the coupling constants are not fixed numbers but vary over some subsets of the reals, for instance for the determination of critical surfaces and universality domains. For every point in coupling constant space, the series construction to 18th order as described above will take a couple of hours on a Sparc Sun work station. For many points this becomes too expensive.

- The 1LI graphs generated are needed for each computation and hence have to be stored. Even in binary coded form this needs a couple of GByte.

- The most severe point is the ill-conditionedness of the composition procedure. If the weights of the graphs involved would be rational (as e.g. for the Ising 
Table 1: The number of inequivalent graphs for the various 1-line irreducible graph classes defined in Section 2. For the first renormalized moments $\mathcal{Q}_{E}(L)$ this number does not depend on $\mathrm{E}$, the number of external lines. For comparison we have given also the number of graphs having only vertices with even number of lines attached. They are denoted by the superscript ${ }^{\mathrm{ev}}$. Notice that $\mathcal{S}_{3}^{\mathrm{ev}}=\emptyset$.

\begin{tabular}{|r|rr|rr|r|}
\hline$L$ & $\mathcal{Q}_{2}^{\text {ev }}(L)$ & $\mathcal{Q}_{2}(L)$ & $\mathcal{S}_{2}^{\text {ev }}(L)$ & $\mathcal{S}_{2}(L)$ & $\mathcal{S}_{3}(L)$ \\
\hline 0 & 1 & 1 & 1 & 1 & 1 \\
1 & 0 & 0 & 0 & 0 & 0 \\
2 & 1 & 1 & 0 & 1 & 1 \\
3 & 0 & 1 & 1 & 1 & 1 \\
4 & 4 & 4 & 0 & 4 & 6 \\
5 & 0 & 6 & 2 & 6 & 12 \\
6 & 15 & 23 & 3 & 23 & 52 \\
7 & 0 & 49 & 8 & 53 & 145 \\
8 & 79 & 174 & 9 & 190 & 567 \\
9 & 0 & 483 & 40 & 575 & 1954 \\
10 & 439 & 1681 & 68 & 2089 & 7577 \\
\hline 11 & 0 & 5446 & 247 & 7349 & 28811 \\
12 & 2877 & 19562 & 470 & 27908 & 115290 \\
13 & 0 & 69575 & 1779 & 106666 & 465112 \\
14 & 20507 & 260752 & 3937 & 423687 & 1930270 \\
\hline 15 & 0 & 989445 & 14801 & 1710920 & 8134945 \\
16 & 161459 & 3874201 & 35509 & 7080691 & 34982052 \\
17 & 0 & 15446928 & 135988 & 29828057 & 152884073 \\
18 & 1376794 & 63028306 & 350614 & 128100002 & \\
\hline
\end{tabular}

model in zero external field), this problem is absent. However, whereas the weights $w(\Gamma)$ are rational numbers, in most cases the vertex couplings $\stackrel{o}{v}_{i}^{c}(\mu)$ are non-rational. They are of both signs, of comparable size, and they are computable to limited precision only. We expect that roundoff errors rapidly accumulate, limiting the series computation above to about the 10th order in the hopping parameter.

A solution to this problem is provided by expansion into vertex structures. Vertex structures have already been introduced and used for $\mathbf{Z}_{2}$ symmetric models [6]. They provide the possibility to arrange the rational parts of the weights according to the precsribed concatenation and recursion relations, keeping track of where and how the vertex couplings $\stackrel{o c}{v}_{i}(\mu)$ are to be multiplied and added. The actual multiplication with the vertices is done only in the very end, when all the other terms 
have been collected, to obtain the final series coefficients of the correlation functions. At this stage then this amounts to a small number of additions and multiplications (a couple of thousands). Hence, using operations on vertex structures, the series construction will become well-conditioned even to large orders. Also, instead of storing the graphs of $\mathcal{S}_{k}$ and $\mathcal{Q}_{k}$, one only needs to keep the vertex structures or their algebraic representations, which are both tiny objects and small in number.

\subsection{Definitions and tadpole moment construction}

In order to work with vertex structures and to understand in which sense they replace graphical manipulations we need to introduce some basic definitions.

A vertex structure $v$ is an ordered sequence of non-negative integers

$$
v=\left(\nu_{i}\right)_{i \in \mathbf{N}}=\left(\nu_{1}, \nu_{2}, \ldots\right) ; \quad \nu_{i} \in\{0,1,2, \ldots\},
$$

with only finitely many $\nu_{i} \neq 0$. For $v$ given by (47) we call $v_{i} \equiv \nu_{i}$ the $i$ th component of $v, s(v) \equiv \max _{v_{i} \neq 0} i$ the size or the length of $v$, and $n(v) \equiv \sum_{i \geq 1} v_{i} i$ the number of lines of $v$. The reason for this becomes clear below where a vertex structure is associated to a graph. We always have $s(v) \leq n(v)$. The set of all vertex structures $v$ with $n(v)=n$ is denoted by $\mathcal{V}_{n}$. We put

$$
\mathcal{V}^{(n)}=\bigcup_{1 \leq k \leq n} \mathcal{V}_{k} .
$$

For non-negative integer $k$ we define the vertex structure $\widehat{k} \in \mathcal{V}_{k}$ by

$$
\widehat{k}=\left(\nu_{i}\right)_{i \in \mathbf{N}}, \text { with } \nu_{k}=1 \text { and } \nu_{i}=0 \text { for } i \neq k .
$$

To a given graph $\Gamma$ a vertex structure $v=v(\Gamma)$ is associated as follows. We set

$$
\begin{aligned}
v(\Gamma) & =\left(\nu_{1}, \nu_{2}, \ldots\right), \\
\nu_{i} & =\left|\left\{u \in \mathcal{B}_{\Gamma} \mid l(u)=i\right\}\right|,
\end{aligned}
$$

that is, $\nu_{i}$ is the number of vertices of $\Gamma$ that have precisely $i$ lines attached. If $\Gamma$ has E external and L internal lines, we have $n(v(\Gamma))=2 L+E$, that is, $v(\Gamma) \in \mathcal{V}_{2 L+E}$.

For given $\Gamma$, the vertex structure $v(\Gamma)$ is uniquely defined, but not vice versa. For given vertex structure $v$ there are many graphs $\Gamma$ such that $v=v(\Gamma)$. Their number is large but finite. This allows us to define a very useful equivalence relation $\sim_{V S}$ in the graph classes under consideration. Hereby, two graphs $\Gamma_{1}$ and $\Gamma_{2}$ are equivalent, $\Gamma_{1} \sim_{V S} \Gamma_{2}$, if $v\left(\Gamma_{1}\right)=v\left(\Gamma_{2}\right)$. This equivalence relation devides the graph classes into small sets of equivalence classes, each class being represented by a single vertex structure. We will associate appropriate rational weights to vertex structures and will define operations on them that are equivalent to the concatenation rules and recursion relations of the last section. 
Toward this end we endow $\cup_{n \geq 1} \mathcal{V}^{(n)}$ with the structure of an associative algebra. First, for later convenience we define the product of two vertex structures $v$ and $v^{\prime}$ by

$$
v \cdot v^{\prime}=\left(v_{i}+v_{i}^{\prime}\right)_{i \in \mathbf{N}} .
$$

By some "external" process, such as the graph association above, a vertex structure gets associated a weight. A pure, weighted vertex structure is a pair $\left(w_{v}, v\right)$ of a number $w_{v}$ of some field $\mathcal{F}$, for our purpose the field of rational numbers, and a vertex structure $v$. For $w_{v}=1$ we use the notation $\mathcal{P}_{v}=(1, v)$ and write $w_{v} \cdot \mathcal{P}_{v} \equiv\left(w_{v}, v\right)$. A weighted vertex structure then is a finite sum of the form

$$
\left(w_{v}\right)_{v \in \mathcal{V}^{(n)}} \equiv \sum_{v \in \mathcal{V}^{(n)}} w_{v} \cdot \mathcal{P}_{v}
$$

This set can be given the structure of an $\mathcal{F}$ vector space in the obvious way, denoted by $\mathcal{W}^{(n)}$. Furthermore, $\mathcal{V}_{k}$ for $1 \leq k \leq n$ become sub-vector spaces of $\mathcal{W}^{(n)}$, which we denote by $\mathcal{W}_{k}$. Finally, with $\mathcal{W}_{0} \equiv \mathcal{F}$,

$$
\mathcal{W}=\bigoplus_{n \geq 0} \mathcal{W}_{n}
$$

is made a graded algebra with unity by defining for $v \in \mathcal{W}_{s}, v^{\prime} \in \mathcal{W}_{t}$ the product $\mathcal{P}_{v} \cdot \mathcal{P}_{v^{\prime}} \equiv \mathcal{P}_{v \cdot v^{\prime}} \in \mathcal{W}_{s+t}$, and by linearity.

It is now easy to translate the series construction into this algebra. We examplify this for the computation of the tadpole moment. First, for the simpler graph classes (the 1LI ones), summation over graphs is replaced by summation over associated vertex structures. This is done by adding the rational weights of all graphs that belong to the same equivalence class of $\sim_{V S}$. For instance, let us define for $L=$ $0,1,2, \ldots$

$$
\widetilde{Q}_{1}(L)=\left\{v \in \mathcal{V}_{2 L+1} \mid v(\Gamma)=v \text { for some } \Gamma \in \mathcal{Q}_{1}(L)\right\} .
$$

For the first renormalized moments with series representation

$$
\chi_{\mathcal{Q}_{1}}=\sum_{L \geq 0}(2 \kappa)^{L} q_{1}(L),
$$

the coefficient of $(2 \kappa)^{L}$ is now obtained as

$$
q_{1}(L)=\sum_{\Gamma \in \mathcal{Q}_{1}(L)} w(\Gamma) \prod_{u \in \mathcal{B}_{\Gamma}} v_{l(u)}^{o c}(\mu)=\sum_{v \in \tilde{Q}_{1}(L)} w_{v}\left(Q_{1}\right) \prod_{i=1}^{s(v)}\left(\stackrel{o c}{c}_{i}^{c}(\mu)\right)^{v_{i}},
$$

where for every vertex structure $v \in \widetilde{Q}_{1}(L)$

$$
w_{v}\left(Q_{1}\right)=\sum_{\Gamma \in \mathcal{Q}_{1}(L) \text { with } v(\Gamma)=v} w(\Gamma) .
$$


The latter sum consists of a large number of terms, but the $w(\Gamma)$ are rational numbers. On the contrary, in (56) the various terms are real numbers only, but their number is very small. With the identification of

$$
w \cdot \prod_{i=1}^{n}\left(\stackrel{\circ}{v}_{i}^{c}(\mu)\right)^{\nu_{i}} \simeq w \cdot \mathcal{P}_{v}, \quad v=\left(\nu_{1}, \nu_{2}, \ldots\right)
$$

$q_{1}(L)$ becomes replaced by

$$
\widetilde{q}_{1}(L)=\sum_{v \in \widetilde{Q}_{1}(L)} w_{v}\left(Q_{1}\right) \cdot \mathcal{P}_{v} \in \mathcal{W}
$$

To the lowest orders, we have e.g. on the $4 \times \infty^{D-1}$ lattice

$$
\begin{aligned}
\widetilde{q}_{1}(0)= & 1 \cdot \mathcal{P}_{(1)} \\
\widetilde{q}_{1}(1)= & 0 \\
\widetilde{q}_{1}(2)= & D \cdot \mathcal{P}_{(0,1,1)} \\
\widetilde{q}_{1}(3)= & \frac{D}{3} \cdot \mathcal{P}_{(0,0,1,1)}, \\
\widetilde{q}_{1}(4)= & \frac{D}{12} \cdot \mathcal{P}_{(0,0,0,1,1)}+\left(6 D^{2}-3 D+1\right) \cdot \mathcal{P}_{(0,3,1)}+D^{2} \cdot \mathcal{P}_{(0,1,1,1)} \\
& +\frac{D^{2}}{2} \cdot \mathcal{P}_{(0,2,0,0,1)},
\end{aligned}
$$

where trailing zeros of the vertex structures have been cut off. The recursion for the series construction of the tadpole moment $\chi_{1}(\kappa, \mu)$, Eqn. (46), is converted into the recursion in the algebra $\mathcal{W}$ as follows. Let us set

$$
\tilde{\chi}_{1}(L=0)=\mathcal{P}_{\widehat{1}},
$$

which is the translation of the initial condition of (46). The analog of (42) becomes

$$
\begin{aligned}
& \tilde{\chi}_{1}^{(1)}(L)=2 D \cdot \widetilde{\chi}_{1}(L-1), \quad L \geq 1, \\
& \tilde{\chi}_{1}^{(\nu)}(L)=\frac{1}{\nu} \cdot \sum_{L^{\prime}=1}^{L-(\nu-1)} \widetilde{\chi}_{1}^{(1)}\left(L^{\prime}\right) \cdot \widetilde{\chi}_{1}^{(\nu-1)}\left(L-L^{\prime}\right), \quad 2 \leq \nu \leq L .
\end{aligned}
$$

The tadpole moment recursion in $\mathcal{W}$ then reads for $L=1,2, \ldots$

$$
\tilde{\chi}_{1}(L)=\sum_{L^{\prime}=0}^{L} \sum_{v \in \widetilde{Q}_{1}\left(L^{\prime}\right)} w_{v}\left(Q_{1}\right) \cdot \prod_{i=1}^{l(v)} \prod_{j=1}^{v_{i}} \sum_{L_{i j}=0}^{L-L^{\prime \prime}}\left(\delta_{L_{i j}, 0} \cdot \mathcal{P}_{\hat{i}}+\sum_{\nu=1}^{L_{i j}} \mathcal{P}_{\hat{i+\nu}} \cdot \tilde{\chi}_{1}^{(\nu)}\left(L_{i j}\right)\right),
$$


where the prime indicates that the summations are restricted to $\sum_{i j} L_{i j}=L-L^{\prime}$. The solution of this recurrence relation yields up to some order $M$, say, the vertex structure representation of $\widetilde{\chi}_{1}(L)$, that is,

$$
\tilde{\chi}_{1}(L)=\sum_{v \in \mathcal{V}_{2 L+1}} w_{v}\left(\chi_{1}\right) \cdot \mathcal{P}_{v}, \quad 0 \leq L \leq M
$$

To the same order as for $\widetilde{q}_{1}$ above we obtain, again on the $4 \times \infty^{D-1}$ lattice,

$$
\begin{aligned}
\tilde{\chi}_{1}(0)= & 1 \cdot \mathcal{P}_{(1)}, \\
\tilde{\chi}_{1}(1)= & 2 D \cdot \mathcal{P}_{(1,1)} \\
\tilde{\chi}_{1}(2)= & D \cdot \mathcal{P}_{(0,1,1)}+4 D^{2} \cdot \mathcal{P}_{(1,2)}+2 D^{2} \cdot \mathcal{P}_{(2,0,1)} \\
\tilde{\chi}_{1}(3)= & \frac{D}{3} \cdot \mathcal{P}_{(0,0,1,1)}+2 D^{2} \cdot \mathcal{P}_{(1,0,2)}+2 D^{2} \cdot \mathcal{P}_{(1,1,0,1)}+2 D^{2} \cdot \mathcal{P}_{(0,2,1)} \\
& +8 D^{3} \cdot \mathcal{P}_{(1,3)}+12 D^{3} \cdot \mathcal{P}_{(2,1,1)}+\frac{4 D^{3}}{3} \cdot \mathcal{P}_{(3,0,0,1)}, \\
\tilde{\chi}_{1}(4)= & \left(6 D^{2}-3 D+1\right) \cdot \mathcal{P}_{(0,3,1)}+\frac{D^{2}}{2} \cdot \mathcal{P}_{(0,2,0,0,1)}+\frac{D}{12} \cdot \mathcal{P}_{(0,0,0,1,1)} \\
& +\frac{2 D^{2}}{3} \cdot \mathcal{P}_{(1,0,0,2)}+\frac{2 D^{2}}{3} \cdot \mathcal{P}_{(1,0,1,0,1)}+6 D^{3} \cdot \mathcal{P}_{(2,0,1,1)}+12 D^{3} \cdot \mathcal{P}_{(1,1,2)} \\
& +8 D^{3} \cdot \mathcal{P}_{(1,2,0,1)}+2 D^{3} \cdot \mathcal{P}_{(2,1,0,0,1)}+\frac{5 D^{2}}{3} \cdot \mathcal{P}_{(0,1,1,1)}+16 D^{4} \cdot \mathcal{P}_{(1,4)} \\
& +48 D^{4} \cdot \mathcal{P}_{(2,2,1)}+\frac{32 D^{4}}{3} \cdot \mathcal{P}_{(3,1,0,1)}+8 D^{4} \cdot \mathcal{P}_{(3,0,2)}+\frac{2 D^{4}}{3} \cdot \mathcal{P}_{(4,0,0,0,1)} .
\end{aligned}
$$

The final series representation of the tadpole moment $\chi_{1}$, (41), is obtained by the map (58), that is,

$$
\chi_{1}(\kappa, \mu)=\sum_{L \geq 0}(2 \kappa)^{L} \chi_{1}(L), \quad \chi_{1}(L)=\sum_{v \in \mathcal{V}_{2 L+1}} w_{v}\left(\chi_{1}\right) \prod_{i=1}^{s(v)}\left(\stackrel{\circ c}{o_{i}^{c}}(\mu)\right)^{v_{i}} .
$$

In a similar way as the computation of the tadpole moments, the vertex renormalization processes are done in terms of vertex structures, that is in the algebra $\mathcal{W}$.

This provides a well conditioned algorithm. To order 18 we have about 5 orders of magnitude less numbers of vertex structures to deal with than we would have graphs. In Table 2 we summarize some numbers on vertex structures.

\section{Summary}

We have generalized the linked cluster expansion to models with nonvanishing vacuum expectation value. The main emphasis here was on the technical details necessary to achieve the hopping parameter series of correlation functions to comparable order as for symmetric models. The major additional problems that had to be solved are the following ones. 
Table 2: The number of mutually different vertex structures associated with various graph classes according to (50), used for the computation of the hopping parameter expansion to order 18. Compared to the number of graphs as given in Table 1, there are about 5 orders of magnitude less number of vertex structures than graphs. $\mathcal{R}_{1}(L)$ denotes the vertex structures of the tadpole moment $\chi_{1}$ to order $L$, that is of the 1-point function $<\rho(x)>$.

\begin{tabular}{|r|r|rr|r|}
\hline$L$ & $\mathcal{Q}_{1}(L)$ & $\mathcal{S}_{2}(L)$ & $\mathcal{S}_{3}(L)$ & $\mathcal{R}_{1}(L)$ \\
\hline 0 & 1 & 1 & 1 & 1 \\
1 & 0 & 0 & 0 & 1 \\
2 & 1 & 1 & 1 & 3 \\
3 & 1 & 1 & 1 & 7 \\
4 & 4 & 3 & 5 & 15 \\
5 & 6 & 5 & 8 & 31 \\
6 & 15 & 12 & 19 & 60 \\
7 & 22 & 19 & 30 & 110 \\
8 & 45 & 37 & 55 & 198 \\
9 & 69 & 58 & 89 & 342 \\
10 & 116 & 103 & 148 & 569 \\
\hline 11 & 183 & 161 & 229 & 943 \\
12 & 294 & 262 & 364 & 1513 \\
13 & 431 & 399 & 551 & 2377 \\
14 & 677 & 628 & 839 & 3700 \\
\hline 15 & 1003 & 938 & 1246 & 5672 \\
16 & 1470 & 1413 & 1838 & 8529 \\
17 & 2148 & 2068 & 2676 & 12745 \\
18 & 3119 & 3047 & & 18775 \\
\hline
\end{tabular}

- The tadpole moment representing the series expansion of the vacuum expectation value $\langle\rho(x)>$ has to be worked out. It obeys a nonlinear recursion relation that involves 1-line irreducible moments only.

- Computation of connected correlation functions requires the attachment of tadpole moments to all vertices. This is conveniently done by including a second vertex renormalization.

- There is a huge proliferation of graphs and in turn of roundoff errors due to the ill-conditioned real arithmetic for generic coupling constants. At the first sight this limits the computations of the series to order 10 in the hopping parameter. The solution of this problem is to replace the graphical expansion of correlation functions and moments by an expansion in terms of weighted vertex structures. The latter can be given the structure of a graded algebra $\mathcal{W}$ with unity over the 
rational numbers. The hopping parameter series construction is done mainly in this algebra.

The vertex structures and their weights have to be computed only once. For the most important moments they are available to 18th order. Using them, for any set of coupling constants (the single site measure (8)), the hopping parameter series themselves are obtained within a couple of minutes on a Sparc Sun workstation.

We have dicussed real valued fields only, merely for simplicity. The ideas behind vertex structures are generalized in a straightforward way to models with larger symmetry group such as $O(N)$, broken down e.g. to $O(N-1)$. This only requires slight changes of equivalence relations and extensions of the algebra $\mathcal{W}$.

Various applications are in preparation and will be presented elsewhere.

\section{References}

[1] M. Wortis, Linked cluster expansion, in Phase transitions and critical phenomena, vol.3, eds. C. Domb and M.S. Green (Academic Press, London 1974).

[2] C. Itzykson and J.-M. Drouffe, Statistical field theory, vol.2, Cambridge University Press, 1989.

[3] A. Pordt, A convergence proof for linked cluster expansions, Univ. Münster preprint MS-TPI-96-05 and e-print archive hep-lat 9604010.

[4] A. Pordt and T. Reisz, Int. Jour. Mod. Phys. A12 (1997) 3739-3757.

[5] M. Lüscher and P. Weisz, Nucl. Phys. B300[FS22] (1988) 325.

[6] T. Reisz, Nucl. Phys. B450 (1995) 569.

[7] T. Reisz, Phys. Lett. 360B (1995) 77.

[8] M. Campostrini, A. Pelissetto, P. Rossi and E. Vicari, Nucl. Phys.. B459 (1996) 207.

[9] S. Zinn, S.-N. Lai and M. E. Fisher, Phys. Rev. E54 (1996) 1176.

[10] P. Butera and N. Comi, Phys. Rev. E55 (1997) 6391.

[11] T. Reisz, "The Gross-Neveu Model and QCDs Chiral Phase Transition", in "Field Theoretical Tools in Polymer and Particel Physics", A. Klümper and H. Meyer-Ortmanns, eds. (Springer Verlag, to appear), and e-print archive heplat 9712017

[12] H. Meyer-Ortmanns and T. Reisz, Jour. Stat. Phys. 87 (1997) 755.

[13] H. Meyer-Ortmanns, K. Pinn and T. Reisz, in preparation. 